\title{
Globally Anisotropic High Porosity Silica Aerogels
}

\author{
J. Pollanen, K. Shirer, S. Blinstein, J.P. Davis, H. Choi, T.M. Lippman, and W.P. Halperin \\ Department of Physics and Astronomy, \\ Northwestern University, Evanston, IL 60208, USA \\ L.B. Lurio \\ Department of Physics, Northern Illinois University, DeKalb, IL 60115, USA
}

(Dated: July 22, 2021)

\begin{abstract}
We discuss two methods by which high porosity silica aerogels can be engineered to exhibit global anisotropy. First, anisotropy can be introduced with axial strain. In addition, intrinsic anisotropy can result during growth and drying stages and, suitably controlled, it can be correlated with preferential radial shrinkage in cylindrical samples. We have performed small angle X-ray scattering (SAXS) to characterize these two types of anisotropy. We show that global anisotropy originating from either strain or shrinkage leads to optical birefringence and that optical cross-polarization studies are a useful characterization of the uniformity of the imposed global anisotropy.

PACS numbers: 61.05.cf, 67.30.hm, 78.20.Fm, 82.70.Gg
\end{abstract}




\section{INTRODUCTION}

High porosity silica aerogel is a transparent, low density, material consisting of aggregates of silica particles, often described as strands, with a diameter of $\sim 30-50 \AA$ and average separation, or correlation length, $\xi \cong 100-1000 \AA[1]$. The microstructure of the aerogel is inherently inhomogeneous on the scale of the correlation length, which we take to be the upper cut-off of a fractal distribution of the silica particles. Fractal distributions follow naturally from the physical process of diffusion limited aggregation that occurs during gel formation and have been characterized with small angle scattering experiments (visible light[2], X-ray[3], and neutrons [4]) as well as having been simulated numerically [5] [6]. In the present work we are particularly interested in long length scale inhomogeneity, specifically the contolled introduction of global anisotropy into silica aerogels and its characterization. In the following we will use the term isotropic to refer to an aerogel having no preferred direction on average over macroscopic specimens that are typically of $\mathrm{mm}$ size or larger.

Silica aerogels with porosities up to $\sim 99 \%$ are of interest in a wide variety of scientific and engineering disciplines including high-energy physics, astrophysics, material science, condensed matter physics and chemical engineering. Aerogels find application in particle physics to detect Cerenkov radiation[7] and have been used by NASA to collect particulate matter from comet 81P/Wild 2[8] 9]. Silica aerogels have seen widespread application in materials science and because of their fractally correlated structure 3 have basic scientific significance. Aerogels are used in the manufacture of ceramic materials [10]. They are important in the study of critical phenomena and exploration of impurity effects in quantum fluids such as ${ }^{4} \mathrm{He}[1]$ and ${ }^{3} \mathrm{He}[12$ as well as to understand the influence of impurities on liquid crystal phase transitions [13] [14]. In addition, hybrid materials based on silica aerogels can be engineered with metal, or metal oxide, particles incorporated into the aerogel framework for the purpose of producing heterogeneous catalysts [15].

Global anisotropy of the aerogel is an important consideration in many of these systems. In general, structural characterization is necessary to understand the behavior of fluids in porous media such as aerogels. This is also true for anisotropic structures which can lead to anisotropic diffusion and flow and therefore be of consequence in many of the aforementioned applications. For example, the ordered phases of liquid crystals in aerogel are inherently anisotropic [13] and we can expect that they will be modified by global anisotropy[16]. The 
physical and optical properties of aerogels are modified by anisotropy, as we discuss in this work, and this may be important in some applications such as transparent thermal insulation[17]. More generally, anisotropic aerogels are members of a larger group of fascinating anisotropic porous media including sedimentary rock[18], bone[19], and cementitious materials[20].

Our motivation for the present study is linked to our interest in a basic physics problem concerning impurity effects on superfluid ${ }^{3} \mathrm{He}$. It is thought that anisotropy will play a role in determining which phases of superfluid ${ }^{3} \mathrm{He}$ in aerogel are energetically stable[21, 22, and that anisotropy will influence the formation of textures of the superfluid order parameter. Here we report that high porosity silica aerogels can be engineered to exhibit global anisotropy and we discuss small angle X-ray scattering (SAXS) and optical polarization methods for characterization of this anisotropy.

\section{SAMPLES}

The aerogel samples discussed in this work were grown at Northwestern University via the "one-step" sol-gel method[23]. A silicon oxide precursor, tetramethyl orthosilicate (TMOS), is dissolved in methanol and hydrolyzed. Ammonia is used as a catalyst.

$$
\mathrm{Si}\left(\mathrm{CH}_{2} \mathrm{OH}\right)_{4}+2 \mathrm{H}_{2} \mathrm{O} \rightarrow \mathrm{SiO}_{2}+4 \mathrm{CH}_{3} \mathrm{OH}
$$

This reaction results in a wet gel, known as alcogel. The alcogel is then supercritically dried following a method that is based on the rapid supercritical extraction process (RSCE) of J.F. Poco et al.[24].

Specifically, the sol is poured into a cylindrical stainless steel chamber having an inner diameter of $5.08 \mathrm{~cm}$ and a length of $4.92 \mathrm{~cm}$ and containing glass tubes of various sizes as molds. The chamber is completely filled with the sol and sealed using two stainless steel lids each having a thickness of $1.27 \mathrm{~cm}$. Each lid is bolted in place using six 8-32 screws. The alcogel that forms is typically aged at room temperature for approximately 3 days. The chamber containing the alcogel is then loaded into an autoclave having an open volume of $0.46 \mathrm{~L}$ and containing $\sim 50 \mathrm{~mL}$ of methanol. The autoclave is loaded into an oven and heated through several manual adjustments and, as the temperature rises, the pressure in the alcogel chamber forces the lids to open slightly, allowing fluid to escape into the open 
volume of the autoclave. Once the critical point of methanol $\left(T_{c}=239.5^{\circ} \mathrm{C}, p_{c}=80.81\right.$ bar) is exceeded the autoclave is emptied by slowly opening a high pressure valve. This drying typically begins from a starting temperature and pressure of $\sim 270^{\circ} \mathrm{C}$ and $\sim 100$ bar. The system is depressurized $\sim 8-12$ hours and then allowed to cool to room temperature overnight. Fig. 1 depicts the typical oven temperature and autoclave pressure profiles during aging and drying. With this method we have successfully produced silica aerogel ranging in

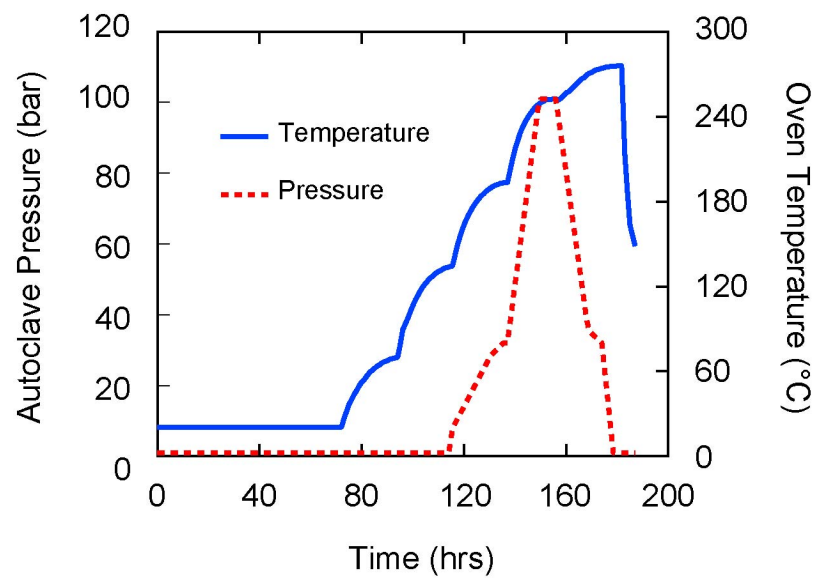

FIG. 1: (Color online) Oven temperature and autoclave pressure profile during aging and drying.

porosity from $\sim 94 \%-99 \%$. A sample's porosity is determined from its weight and volume after supercritical drying to an accuracy of $0.1 \%$ for a $98 \%$ aerogel.

For the SAXS and optical birefringence measurements reported here, cylindrical samples with porosities ranging from $\sim 97 \%-98 \%$ were grown in glass tubes with $\sim 8$ mm inside diameter. By controlling the catalyst concentration and the supercritical drying procedure we could grow samples with radial shrinkage in the range $0 \%$ to 20\%. From these samples we chose those with no significant shrinkage on-axis. The samples were cut to $\sim 0.75 \mathrm{~cm}$ with aspect ratios of $\sim 1$.

\section{SAXS EXPERIMENTS}

The scattering experiments were carried out at Sector 8 of the Advanced Photon Source (APS) at Argonne National Laboratory, using a photon energy of $7.5 \mathrm{keV}$, corresponding to a wavelength, $\lambda=1.69 \AA$. The X-ray beam, with a spot size of $\approx 100$ microns, was incident on the the center of a sample, which was oriented with its cylinder axis perpendicular to 
the beam. The experiments discussed below can be grouped into two categories. First, to study the effect of axial strain on the aerogel, scattering experiments were carried out on a nominally isotropic sample as well as on a sample exhibiting $13.8 \%$ radial shrinkage. They were subjected to increasing strain in the range $0 \%$ to $\sim 32 \%$. Secondly, in order to systematically explore the relationship between radial shrinkage and X-ray scattering, we conducted SAXS on a series of samples exhibiting radial shrinkage in the range $0 \%$ to $20 \%$.

In each experiment we recorded the scattered X-ray intensity, $I(q)$, as a function of the momentum transfer, $q$, using a CCD camera. The momentum transfer is defined as $q=$ $(4 \pi / \lambda) \sin (\theta / 2)$, where $\theta$ is the angle between the incident and scattered X-ray wavevectors. For a given image, we binned $I(q)$ in $\sim 10^{\circ}$ increments of the azimuthal angle, $\phi$, defined in the plane of the CCD camera (refer to Fig. 2). The cylinder axis was defined to be $\phi=90^{\circ}$. Fig. 2 is a cartoon depicting the geometry of a typical SAXS measurement.

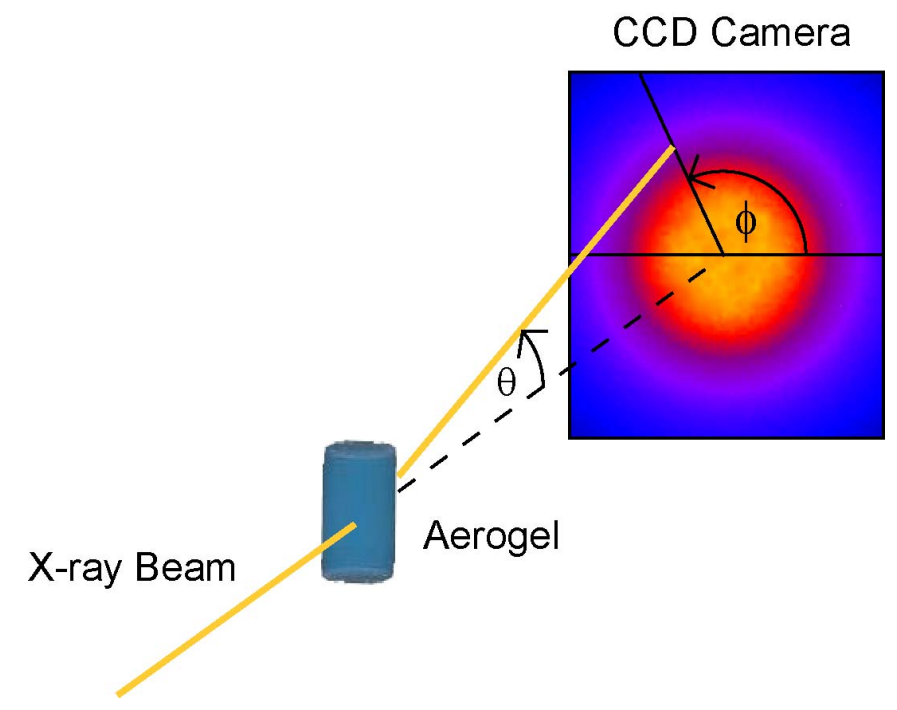

FIG. 2: (Color online) Sketch of the experimental geometry. Note that $\phi=90^{\circ}$ is parallel to the cylinder axis.

\section{SAXS RESULTS AND DISCUSSION I: AXIAL STRAIN AND ANISOTROPY}

The dependence of $I(q, \phi)$ on $\phi$ provides information about the magnitude and direction of anisotropy in the aerogel. This is depicted in Fig. 3, which presents images of the scattered X-ray intensity from two aerogel samples. The sample labeled (a) is of a nominally isotropic and unstrained aerogel, while $\left(\mathrm{a}^{\prime}\right)$ depicts the same sample, but strained by $12.7 \%$ along the 
cylinder axis. The image labeled (b) is of a sample exhibiting $13.8 \%$ radial shrinkage. In what follows, the labeling scheme presented in Fig. 3 will be used when referring to these two samples. The porosity of sample (a) was $98 \%$ and that of (b) $97.6 \%$. Fig. 3 qualitatively

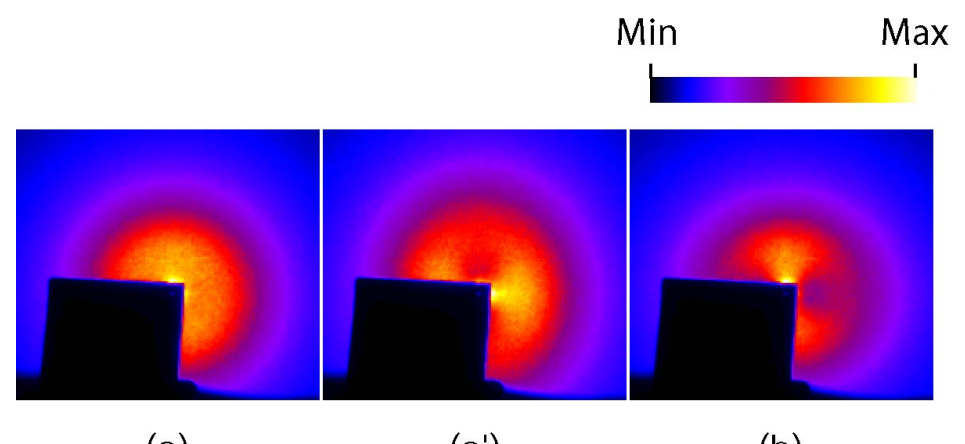

(a)

$\left(a^{\prime}\right)$

(b)

FIG. 3: (Color online) False color scattered X-ray intensities for (a) a $98 \%$ nominally isotropic aerogel, $\left(\mathrm{a}^{\prime}\right)$ the same sample unaxially strained by $12.7 \%$, and (b) a $97.6 \%$ aerogel exhibiting $13.8 \%$ radial shrinkage. In each case the sample is oriented with its cylinder axis vertical relative to the images. The dark rectangle is a beamstop inserted to protect the CCD camera from the unscattered transmitted beam.

demonstrates the existence of two distinct types of anisotropy. First, comparing (a) and $\left(a^{\prime}\right)$ shows that axial strain can be used to introduce anisotropy into a nominally isotropic aerogel. In addition, (b) indicates the presence of intrinsic anisotropy in samples exhibiting radial shrinkage. Moreover, this intrinsic anisotropy is $\sim 90^{\circ}$ out of phase with that induced by axial strain.

We analyzed the scattering curves by fitting $I(q, \phi)$ with the following phenomenological scattering function,

$$
I(q, \phi)=\frac{C \xi^{d}}{\left(1+q^{4} \xi^{4}\right)^{d / 4}} \frac{\left(1+q^{2} \xi^{2}\right)^{1 / 2}}{q \xi} \sin \left[(d-1) \tan ^{-1}(q \xi)\right],
$$

where $C, d$, and $\xi$ are fit parameters which depend on $\phi$. Eq. 1 is a modified version of a structure factor for fractally correlated materials having an upper length scale cut-off. The $q$-dependence of Eq. 1 is stronger than the structure factor derived by Freltoft et. al. [25]. This sharper $q$-dependence was chosen to better match our experimental data. The parameter $d$ is the fractal dimension of the aerogel, and $C$ is an overall scale factor. The length scale $\xi$ is associated with the aerogel correlation length, i.e. the upper length scales at which the aerogel ceases to be fractally correlated and, for future reference, we note that 
the axial (radial) correlation length corresponds to $\phi=90^{\circ}\left(\phi=0^{\circ}\right)$. Note that in the limit $q \xi \gg 1$, Eq. 1 is proportional to $q^{-d}$ as expected [25] for the fractal regime. We performed fits to our data using Eq. 1 and obtained an excellent representation as can be seen in a typical case in Fig. 4 for sample $\left(\mathrm{a}^{\prime}\right)\left(\phi=5.3^{\circ}, \phi=88.5^{\circ}\right)$. Fig. 4 shows that when the aerogel is compressed along the cylinder axis the axial correlation length is compressed relative to the correlation length in the radial direction.

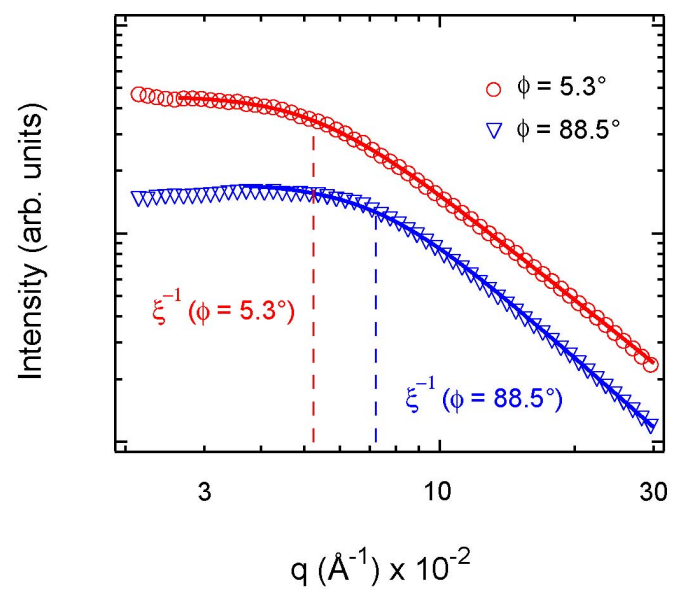

FIG. 4: (Color online) $I(q, \phi)$ fit with Eq. $1\left(\phi=5.3^{\circ}, \phi=88.5^{\circ}\right)$ for the sample $\left(\mathrm{a}^{\prime}\right)$. The curves have been offset vertically for clarity, otherwise the data points would coincide at high $q$.

To quantify the magnitude and direction of the anisotropy in a given sample, we plot the correlation length $\xi$ versus $\phi$. For all of the samples we found the following $\phi$-dependence,

$$
\xi(\phi)=\xi_{0}+\xi_{1} \cos (2 \phi)
$$

where $\xi_{0}$ and $\xi_{1}$ are fit parameters. Note, the average fractal dimension ranged from 1.55-1.80 over the samples discussed in this work. For any given sample, the fractal dimension, $d$, was found to be a much weaker function of $\phi$, as compared to $\xi(\phi)$. The dependences of $d$ and $C$ on $\phi$ are not important to our analysis and do not pertain to our discussion. The parameter $\xi_{0}$ represents the average correlation length of the aerogel and was found to be of the order of $\sim 200 \AA$. The amplitude, $\xi_{1}$, is a measure of the magnitude of the anisotropy. In Fig. 5 we present this $\phi$-dependence for the samples (a), ( $\left.a^{\prime}\right)$, and (b). The sinusoidal dependence of $\xi(\phi)$ demonstrates that anisotropy from axial strain, or radial shrinkage, exists on the length scale of the aerogel correlation length, $\xi$. Moreover, comparing the traces for samples $\left(a^{\prime}\right)$ and (b) demonstrates that the axial strain induced anisotropy has the opposite sign of 


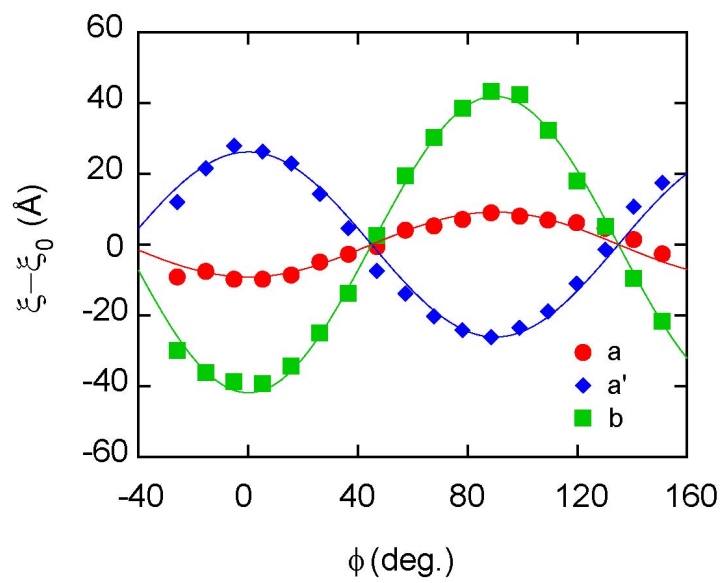

FIG. 5: (Color online) $\xi-\xi_{0}$ as a function of $\phi$ for the samples (a), (a'), and (b). The solid curves are fits using Eq. 2. The amplitude of the sinusoid is a measure of the magnitude of anisotropy.

that from anisotropy produced by radial shrinkage. Fig. 5 also indicates that the nominally isotropic sample, i.e. sample (a), also possesses a small amount of intrinsic anisotropy, which has a magnitude $\xi_{1} \simeq 9 \AA$.

To further investigate the relationship between axial strain and global anisotropy we plot $\xi_{1}$ versus applied axial strain. The results for samples (a) and (b) are presented in Fig. 6. The increase of $\xi_{1}$ with increasing strain demonstrates that anisotropy can be introduced

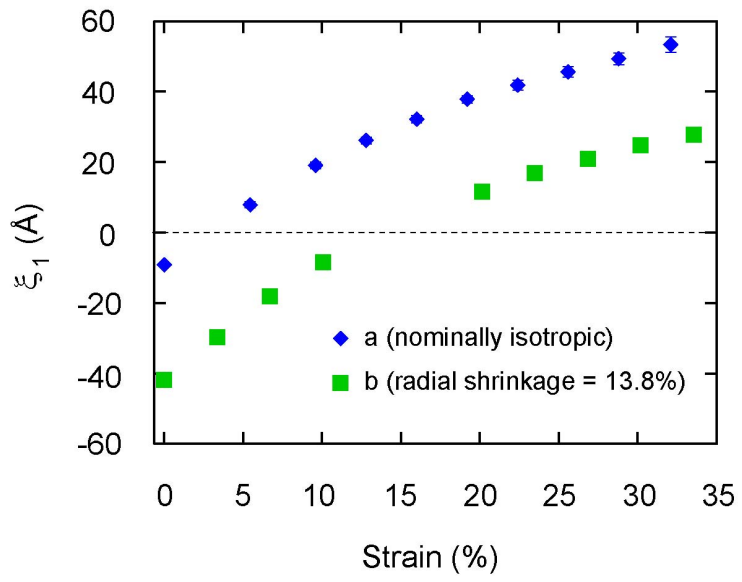

FIG. 6: (Color online) $\xi_{1}$ as a function of axial strain applied to samples (a) and (b). The vertical offset between the two data sets reflects the difference in magnitude of the intrinsic anisotropies of the two samples. 
into the aerogel systematically. Moreover, axial strain can be used to tune between the two types of anisotropy and implies that strain can be used to compensate for intrinsic anisotropy. The negative values of $\xi_{1}$ are an indication of the overall phase in the second term in Eq. 2.

We also plot $\xi_{0}$ versus strain to see how the average correlation length of a sample is affected. The results for samples (a) and (b) are presented in Fig. 7. We find that $\xi_{0}$ decreases monotonically with applied strain for both samples. Previous SAXS studies on isostatically strained aerogels also indicate a decrease in the correlation length with applied strain[26].

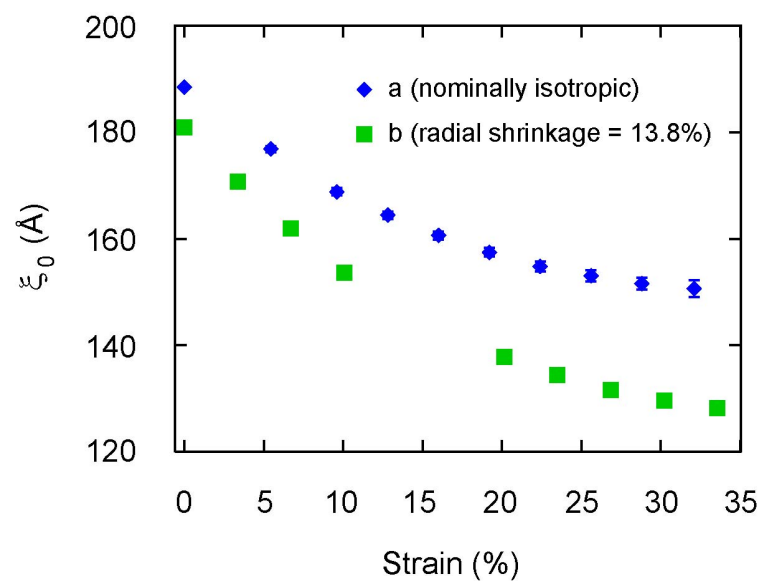

FIG. 7: (Color online) $\xi_{0}$ as a function of axial strain applied to samples (a) and (b).

Fig. 6 and Fig. 7] seem to suggest that the primary effect of strain is to reduce the axial correlation length while only weakly increasing the radial correlation length. The correlation length parallel (perpendicular) to the cylinder axis can be obtained from Eq. 2 by setting $\phi=90^{\circ}\left(\phi=0^{\circ}\right)$ and we define $\xi_{\|} \equiv \xi_{0}-\xi_{1}\left(\xi_{\perp} \equiv \xi_{0}+\xi_{1}\right)$ as the axial (radial) correlation length. In Fig. 8 we plot $\xi_{\|}$and $\xi_{\perp}$ divided by their values at zero strain, $\xi_{\| 0}$ and $\xi_{\perp 0}$, versus strain applied to samples (a) (uppper panel) and (b) (lower panel). Fig. 8 clearly demonstrates that axial strain primarily compresses $\xi_{\|}$, i.e. the correlation length parallel to the strain axis, while only modestly increasing $\xi_{\perp}$.

Fig. 8 also allows us to determine the extent to which the macroscopic strains are transmitted to microscopic length scales, i.e. length scales of the order of $\xi$. For small values of 

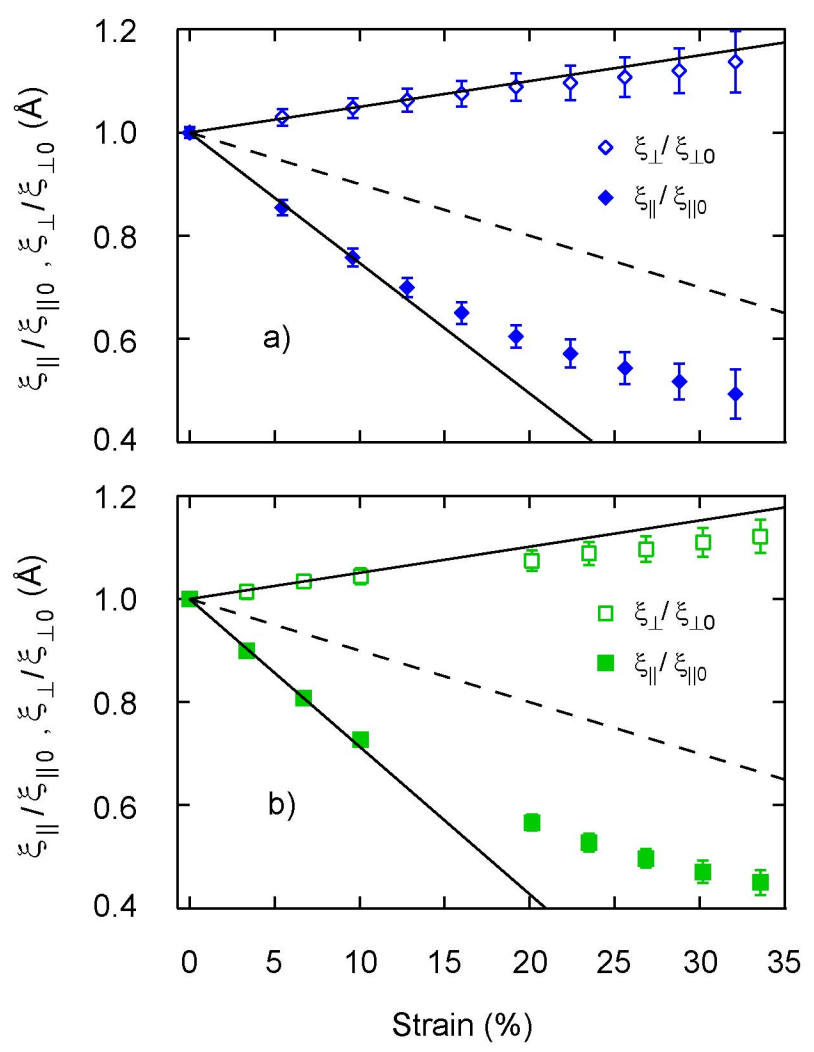

FIG. 8: (Color online) Normalized axial (radial) correlation length, $\xi_{\|} / \xi_{\| 0}\left(\xi_{\perp} / \xi_{\perp 0}\right)$, versus strain for sample (a) (upper panel) and (b) (lower panel). The solid lines are linear fits to the low strain portion of $\xi_{\|} / \xi_{\| 0}$ and $\xi_{\perp} / \xi_{\perp 0}$. The dashed line corresponds to $\alpha=1$ in Eq. 3 and 4 .

strain the data in Fig. 8 indicate,

$$
\begin{gathered}
\frac{\xi_{\|}}{\xi_{\| 0}}=\left(1+\alpha \epsilon_{\|}\right) \\
\frac{\xi_{\perp}}{\xi_{\perp 0}}=\left(1-\beta \epsilon_{\|}\right),
\end{gathered}
$$

where $\epsilon_{\|} \equiv \triangle l / l_{0}$ is the axial strain on a cylindrical aerogel intially having a length of $l_{0}$ and compressed by $\triangle l$. The constants $\alpha$ and $\beta$ describe the transmission of macroscopic strain down to the level of the correlation length and are determined from linear fits of $\xi_{\|} / \xi_{\| 0}$ and $\xi_{\perp} / \xi_{\perp 0}$ near zero strain. These fits are presented in Fig. 8 as solid lines for samples (a) (upper panel) and (b) (lower panel). In Eq. 3 and 4 we use the convention that $\epsilon_{\|}<0$ for compression. Homogeneous elastic theory (HET) suggests that $\alpha=1$ and $\beta=\nu$, where $\nu$ is the macroscopic Poisson ratio. Note, $\alpha=1$ implies that the macroscopic strain scales directly down to the level of the aerogel correlation length and is represented by the dashed 
lines in Fig. 8. If we had found $\alpha<1$ it would suggest that strain is not distributed to microscopic length scales, while $\alpha>1$ would imply an enhancement at microscopic length scales. The dependence of $\xi_{\|} / \xi_{\| 0}$ on strain in Fig. 8 unambiguously indicates a value of $\alpha>1$, implying that the aerogel cannot be accurately described by HET. We find $\alpha=2.5 \pm 0.1$ for sample (a) and $\alpha=2.9 \pm 0.1$ for sample (b). However, from our measurements we find that the ratio $\beta / \alpha \approx \nu$. Specifically $\beta / \alpha=0.20 \pm 0.01$ for sample (a) and $\beta / \alpha=0.18 \pm 0.02$ for sample (b). These values are qualitatively consistent with typical values of $\nu$ for silica aerogels[27]. In addition, we have measured the macroscopic Poisson ratio for a sample similar to (a) using optical techniques (see section VII) and find $\nu=0.30 \pm 0.05$.

We conclude that aerogel is not a homogenous elastic medium and that axial strain induces larger effects on the correlation length scale than would be expected for such a medium. Finally, a Poisson-like behavior describing the microscopic displacements appears to hold.

\section{SAXS RESULTS AND DISCUSSION II: INTRINSIC ANISOTROPY AND RA- DIAL SHRINKAGE}

To explore the possibility of systematically introducing anisotropy different from that produced by strain, we have also performed SAXS on a series of aerogels which exhibited preferential radial shrinkage after supercritical drying. Following the same analysis as detailed in the previous section, we studied the evolution of the the anisotropy amplitude, $\xi_{1}$, as a function of radial shrinkage. The results are presented in Fig. 9. Specifically, the absolute value of $\xi_{1}$ appears to increase systematically with increasing shrinkage, indicating a correlation between radial shrinkage and anisotropy. The larger data scatter in Fig. 7 as compared with Fig. 5, reflects that our measurement and control of radial shrinkage is not as uniform and systematic as for imposed strain on a single sample. However, the small anisotropy of the samples having small shrinkage is prominent. It is noteworthy that the magnitude of the effect on $\left|\xi_{1}\right|$ is comparable with that observed for strained samples.

In addition, we plot the average correlation length, $\xi_{0}$, as a function of radial shrinkage in Fig. 10. For the samples that exhibited radial shrinkage, $\xi_{0}$ was found to decrease by as much as $\sim 40 \%$. This effect is of similar magnitude as that observed for strained samples with the exception of the samples that did not show any shrinkage and which are not consistent with 


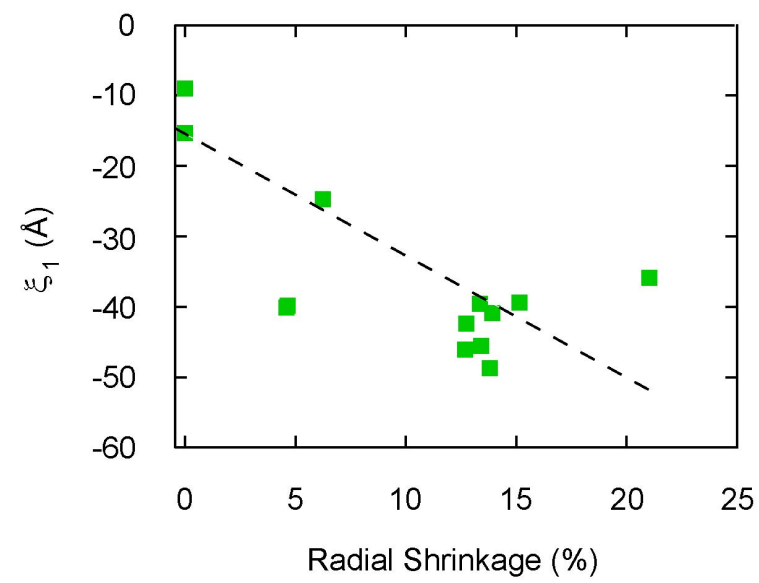

FIG. 9: (Color online) Anisotropy amplitude, $\xi_{1}$, as a function of radial shrinkage. The dashed line is a guide to the eye. Note the relatively small magnitude of intrinsic anisotropy in the samples that did not exhibit shrinkage.

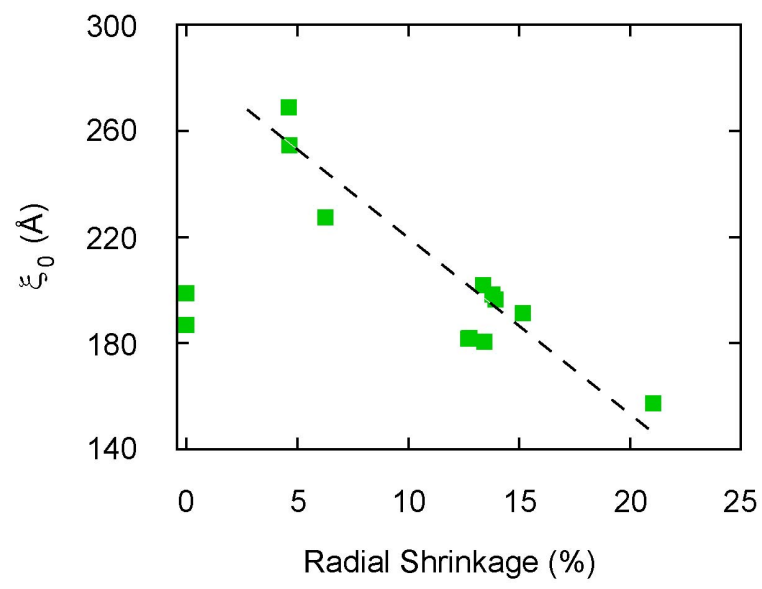

FIG. 10: (Color online) Average correlation length, $\xi_{0}$, as a function of radial shrinkage. The dashed line is a guide to the eye. Note, the behavior of the samples exhibiting no shrinkage is inconsistent with the general trend presented here, for which we have no explanation.

the the general trend in Fig. 10, for reasons we do not understand.

\section{OPTICAL BIREFRINGENCE EXPERIMENTS}

When a transparent material possesses an anisotropic dielectric constant it will exhibit birefringence. Illuminating an optically birefringent material with white light will result in transmission of two components with orthogonal polarizations, parallel and perpendicular 
to the "optical axis". By placing such a material between crossed polarizers it is possible to determine if there is a well defined optical axis and how it is distributed throughout the sample[28]. Measurements of optical birefringence are well-known for characterization of liquid crystals [29] and many other materials [30].

We have performed optical birefringence experiments on high porosity aerogels. Cylindrical aerogel samples were placed between two crossed polarizers and illuminated with diffuse white light. The cylinder axis was oriented vertically, a geometry similar to that shown in Fig. 1. A diffuser was placed between the light source and the first polarizer and images were recorded with a digital camera located after the second polarizer.

To study the effect of axial strain on a nominally isotropic aerogel, optical birefringence experiments were conducted as the sample was subjected to increasing strain in the range $0 \%$ to $18.6 \%$. In addition, we have investigated samples with radial shrinkage and have confirmed that optical birefringence can be used to detect global anisotropy in this situation as well. In both cases, the optical axis is primarily oriented along the cylinder axis.

\section{OPTICAL BIREFRINGENCE RESULTS AND DISCUSSION}

Fig. 11 presents optical birefringence of a $98 \%$ porosity aerogel as it was axially strained to $18.6 \%$ in $2.3 \%$ steps. The crossed polarizers were oriented with one polarizer at $45^{\circ}$ and the other at $135^{\circ}$ relative to the vertical cylinder axis. This sample did not exhibit any radial shrinkage after supercritical drying. Panel 1 of Fig. 11 indicates that the unstrained gel is

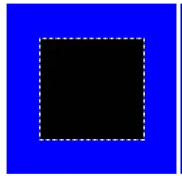

1

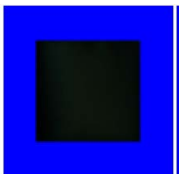

2

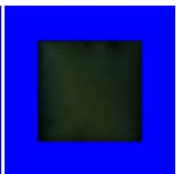

3

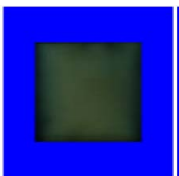

4

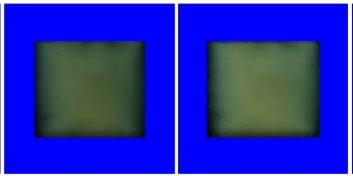

5

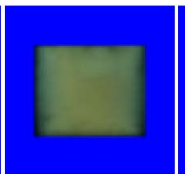

7

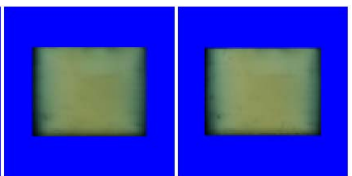

8
9

FIG. 11: (Color online) Optical birefringence of a 98\% porosity aerogel, nominally isotropic before it was subjected to increasing axial strain. The strain increases from left to right: 1 (unstrained), $2(2.3 \%$ strain), 3 (4.7\%), 4 (7.0\%), 5 (9.3\%), 6 (11.6\%), 7 (14.0\%), 8 (16.3\%), 9 (18.6\%).

homogeneously isotropic since it does not impose any preferred direction of polarization when placed between crossed polarizers for all orientations of the polarizers with respect to the cylinder axis. We have also found no transmission of light propagating down the 
cylinder axis for this isotropic aerogel placed between crossed polarizers. Panels 2-9 of Fig. 11 demonstrate that strain can be used to effectively convert this nominally isotropic aerogel into a polarizer. We attribute this effect to optical birefringence. The polarizing effect increases with increasing strain and is the result of structural anisotropies on optical length scales. Furthermore, it is evident from the approximately uniform intensity of the image that this anisotropy is a global property of the entire sample and is homogeneously distributed. From the images presented in Fig. 11 we can measure the relative intensity of light passing through the sample averaged over a central region of the image of the aerogel as a function of strain. These results are presented in Fig. 12 and indicate that the transmitted intensity increases linearly with increasing strain up to the largest value of strain, i.e. $18.6 \%$.

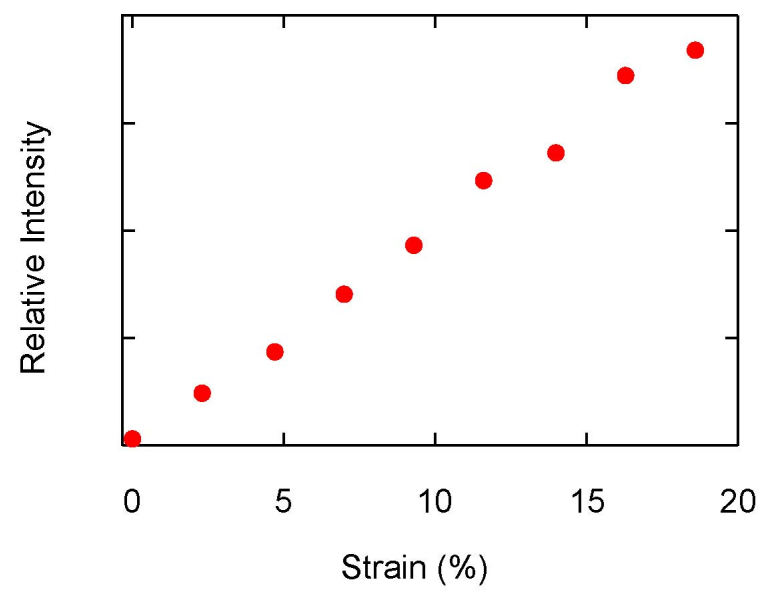

FIG. 12: (Color online) Relative intensity averaged over a central region of the image of the sample versus strain for the sample presented in Fig. 11 .

By rotating both polarizers, keeping them crossed, we can determine the direction of the anisotropy axis. Fig. 13 presents such a rotation sequence for the sample at it's maximum compression, i.e. $18.6 \%$. The fact that the intensity maxima (minima) are seen when the polarizers are oriented at $45^{\circ}$ and $135^{\circ}\left(90^{\circ}\right.$ and $\left.180^{\circ}\right)$ is consistent with the anisotropy axis being oriented along the cylinder (strain), axis.

To complement our SAXS measurements, we also used optical birefringence to investigate possible intrinsic anisotropy in a sample exhibiting radial shrinkage. As before, the polarizers were intially oriented with one at $45^{\circ}$ and the other at $135^{\circ}$ relative to the cylinder axis. They were then rotated together, keeping them crossed. We have found that aerogels exhibiting 


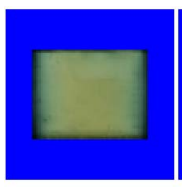

1

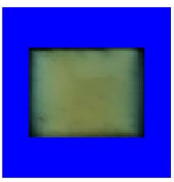

2

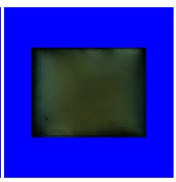

3

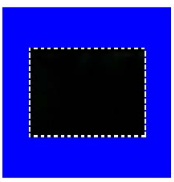

4

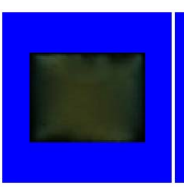

5

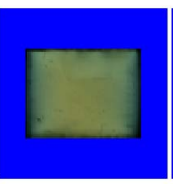

6

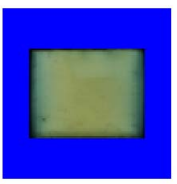

7

FIG. 13: (Color online) Optical birefringence of a $98 \%$ porosity aerogel strained by $18.6 \%$. The labels are associated with the rotation of the polarizers relative to the cylinder axis: $1\left(45^{\circ}, 135^{\circ}\right)$, $2\left(60^{\circ}, 150^{\circ}\right), 3\left(75^{\circ}, 165^{\circ}\right), 4\left(90^{\circ}, 180^{\circ}\right), 5\left(105^{\circ}, 195^{\circ}\right), 6\left(120^{\circ}, 210^{\circ}\right), 7\left(135^{\circ}, 225^{\circ}\right)$.

radial shrinkage polarize transmitted light and this can be observed with cross polarizers, indicating the existence of a well defined optical axis. Again we attribute this effect to optical birefringence. In Fig. 14 we present the results for an aerogel cylinder exhibiting $12.7 \%$ radial shrinkage showing that axial intrinsic anisotropy is present in this sample. It is noteworthy that this intrinsic anisotropy seems to be less uniform throughout the sample than that induced by strain as displayed in panels 2, 3, 5, and 6 of Fig. 14 .

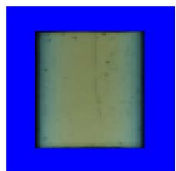

1

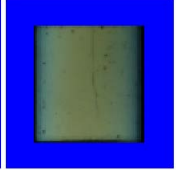

2

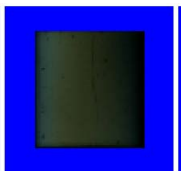

3

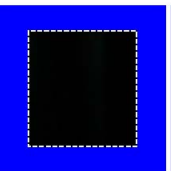

4

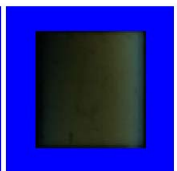

5

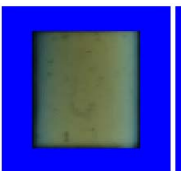

6

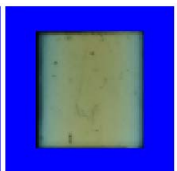

7

FIG. 14: (Color online) Optical birefringence of a 98\% porosity aerogel exhibiting 12.7\% radial shrinkage. The labels are associated with the rotation of the polarizers relative to the cylinder axis: $1\left(45^{\circ}, 135^{\circ}\right), 2\left(60^{\circ}, 150^{\circ}\right), 3\left(75^{\circ}, 165^{\circ}\right), 4\left(90^{\circ}, 180^{\circ}\right), 5\left(105^{\circ}, 195^{\circ}\right), 6\left(120^{\circ}, 210^{\circ}\right), 7\left(135^{\circ}\right.$, $\left.225^{\circ}\right)$

For the intrinsically anisotropic samples, i.e. those exhibiting radial shrinkage, we observed only a small amount of transmission through crossed polarizers for light propagating down the cylinder axis. This, along with the rotation series presented in Fig. 14, indicates that the cylinder axis is predominantly the optical axis in the case of samples exhibiting anisotropy due to radial shrinkage.

Our SAXS measurements indicate that microscopic anisotropy is associated with strain or radial shrinkage developed during the sample growth and drying stages. Furthermore, with a SAXS spot size of $\approx 100$ microns it is possible to image the distribution of anisotropy by scanning the sample profile. We have found that tabletop optical polarization studies 
are complementary and less time consuming. They give a clear picture of the principal directions of the anisotropy on optical length scales and have the advantage of displaying an image that shows the homogeneity of this anisotropy as it is distributed over the sample.

\section{CONCLUSIONS}

In conclusion, our measurements demonstrate that high porosity silica aerogels can be engineered with global structural anisotropy produced either by applied strain or during sample growth and drying. SAXS measurements indicate that this anisotropy is on the length scale of the aerogel correlation length, $\xi$, and optical birefringence measurements confirm that it is a global feature of the aerogel. These aerogels represent a novel type of anisotropic porous medium with possible implications for applications in a wide variety of physical systems.

\section{Acknowledgments}

We would like to thank J.A. Sauls for valuable theoretical insights. We are grateful to N. Mulders, G.W. Scherer, and J.F. Poco for their advice regarding aerogel shrinkage and fabrication. We are indebted to P.E. Wolf and F. Bonnet for introducing us to the technique of optical polarization studies. B. Reddy and W.J. Gannon have provided many useful discussions.

This work was supported by the National Science Foundation, DMR-0703656. Use of the Advanced Photon Source was supported by the U. S. Department of Energy, Office of Science, Office of Basic Energy Sciences, under Contract No. W-31-109-ENG-38.

[1] J. Fricke, Scientific American 258, No. 5, 92 (1988).

[2] A.J. Hunt and P. Berdahl, Mater. Res. Soc. Symp. Proc. 32, 275 (1984).

[3] D.W. Schaefer and K.D. Keefer, Phys. Rev. Lett. 56, 2199 (1986).

[4] R. Vacher, T. Woignier, and J. Pelous, Phys. Rev. B 37, 6500 (1988).

[5] A. Hasmy, R. Vacher, R. Jullien, Phys. Rev. B 50, 1305 (1994).

[6] T.A. Witten and L.M. Sander, Phys. Rev. Lett. 47, 1400 (1981). 
[7] M. Cantin, M. Casse, L. Koch, R. Jouan, P. Mestreau, D. Roussel, C.Saclay, F. Bonnin, J. Moutel, S.J. Teichner, Nucl.Instrum. Methods, 118, 177 (1974).

[8] J. Baker, Science, 314 (5806), 1707 (2006).

[9] D. Brownlee, et al., Science, 314 (5806), 1711 (2006).

[10] L.W. Hrubesh, T.M. Tillotson, and J.F. Poco, in Better Ceramics Through Chemistry IV, ed. by B.J.J. Zelinski, C.J. Brinker, D.E. Clark, and D.R. Ulrich, MRS Symposia Proceedings No. 180 (Materials Research Society, Pittsburgh), 315 (1990).

[11] M. Chan, N. Mulders, and J. Reppy, Physics Today, 8, 30 (1996).

[12] W.P. Halperin and J.A. Sauls, Helium-Three in Aerogel, arXiv:cond-mat/0408593v1.

[13] Liquid Crystals in Complex Geometries, ed. G. P. Crawford and S. Žumer (Taylor and Francis, London) (1996).

[14] N.A. Clark, T. Bellini, R.M. Malzbender, B.N. Thomas, A.G. Rappaport, C.D. Muzny, D.W. Schaefer, and L. Hrubesh, Phys. Rev. Lett. 71, 3505 (1993).

[15] J.B. Miller, S.E. Rankin, and E.I. Ko, Journal of Catalysis, 148, 673 (1994).

[16] B. Jacobsen, K. Saunders, L. Radzihovsky, and J. Toner, Phys. Rev. Lett. 83, 1363 (1999).

[17] A. Emmerling, R. Petricevic, A. Beck, P. Wang, H. Scheller, J. Fricke, Journal of NonCrystalline Solids, 185, Number 3, 240-248(9) (1995).

[18] A.M. Winkelmolen, Sedimentology, 29, 255-256 (1982).

[19] J. Phelps, J. Shields, X. Wang, and C.M. Agrawal, in Proceedings of the 17th Southern Biomedical Engineering Conference, 23 (1998).

[20] S. Mindess, J.F. Young, Concrete, 439, (Pretence Hall Inc., Englewood Cliffs, NJ).

[21] K. Aoyama and R. Ikeda, Phys. Rev. B. 73, 060504(R) (2006).

[22] J.P. Davis, H. Choi, J. Pollanen, and W.P. Halperin, AIP Conf. Proc. 850, 239 (2006).

[23] S.J Teichner, Aerogels of Inorganic Oxides, in Aerogels: Proceedings of the first international symposium, Würzburg, Fed. Rep. of Germany, September 23-25, 1985, ed. by J. Fricke 22-30 (Springer-Verlag Publishers, Berlin, New York) (1985).

[24] J.F. Poco, P.R. Coronado, R.W. Pekala, L.W. Hrubesh, in: R.F. Lobo, J.S. Beck, S.L. Suib, D.R. Corbin, M.E. Davis, L.E. Iton, S.I. Zones (Eds.), Microporous and Mesoporous Materials, MRS Symposium Proceedings, 431, Materials Research Society, Pittsburg, PA, 297 (1996).

[25] T. Freltoft, J.K. Kjems, and S.K. Sinha, Phys. Rev. B 33, 269 (1986).

[26] T. Woignier, I. Beurroies, P. Delord, V. Gibiat, R. Sempere, and J. Phalippou, Eur. Phys. J. 
AP 6, 267 (1999).

[27] J. Gross, G. Reichenauer, and J. Fricke, J. Phys. D: Appl. Phys 21, 1447 (1988).

[28] E. Hecht, Optics 3rd ed., 330, (Addison-Wesley, Massachusetts) (1998).

[29] G.W. Gray, Molecular Structure and the Properties of Liquid Crystals, (Academic, New York) (1962).

[30] D.A. Balzarini, Phys. Rev. Lett. 25, 914 (1970). 